\title{
Human responses to Covid-19: The role of optimism bias, perceived severity, and anxiety
}

\author{
Iro Fragkaki $^{\text {a, }}$, Dominique F. Maciejewski ${ }^{\mathrm{a}}$, Esther L. Weijman ${ }^{\mathrm{a}}$, Jonas Feltes ${ }^{\mathrm{b}}$, Maaike Cima ${ }^{\mathrm{a}}$ \\ ${ }^{a}$ Behavioural Science Institute, Radboud University, Motenssorilaan 3, 6525 HR, Nijmegen, the Netherlands \\ ${ }^{\mathrm{b}}$ Faculty of Technology, Policy and Management, Delft University of Technology, Mekelweg 5, 2628 CD Delft, the Netherlands
}

\section{A R T I C L E I N F O}

\section{Keywords:}

Covid-19

Optimism bias

Anxiety

Perceived severity

Behavioral changes

\begin{abstract}
A B S T R A C T
During the Covid-19 pandemic, the governments are trying to contain the spread with non-pharmaceutical interventions (NPIs), such as social distancing rules, restrictions, and lockdowns. In an effort to identify factors that may influence population adherence to NPIs, we examined the role of optimism bias, anxiety, and perceived severity of the situation in relation to engagement in protective behavioral changes and satisfaction with governments' response to this pandemic. We conducted an online survey in 935 participants $\left(M_{\text {age }}=34.29 ; 68.88 \%\right.$ females) that was disseminated in April and May 2020 in the Netherlands, Germany, Greece, and USA. Individuals with high optimism bias engaged less in behavioral changes, whereas individuals with high levels of anxiety and high perceived severity engaged more in behavioral changes. Individuals with high optimism bias and high levels of anxiety were less satisfied with the governments' response, albeit for different reasons. Individuals who reported low perceived severity and low government satisfaction engaged the least in behavioral changes, whereas participants who reported high perceived severity and low government satisfaction engaged the most in behavioral changes. This study contributes to a better understanding of the psychological factors that influence people's responses to NPIs.
\end{abstract}

\section{Introduction}

The new coronavirus SARS-CoV-2 has led to a pandemic of the Covid-19 disease and most countries are currently trying to contain the spread with non-pharmaceutical interventions (NPIs). NPIs include measures such as social distancing, school/business closures, quarantine, and lockdowns. NPIs were consistently applied during previous pandemics and were effective in containing the spread of viruses, reducing the peak of infections, and minimizing the burden on health care systems (Balinska \& Rizzo, 2009). During the current pandemic, countries around the globe have imposed different NPIs with various levels of effectiveness (Hale, Webster, Petherick, Phillips, \& Kira, 2020).

The most crucial factor in the effectiveness of NPIs during a pandemic is population adherence. A review of evidence from NPIs during previous pandemics has revealed several factors associated with higher compliance with the imposed measures (Bish \& Michie, 2010). Particularly, educated females, non-Caucasians, older people, as well as people with higher perceived risk of infection, higher levels of anxiety, higher perceived severity of the disease, and higher confidence in the effectiveness of the measures tend to show higher compliance with NPIs.

To explain and understand how people react to a health threat, several theories of behavior have been developed, such as the health belief model, the theory of planned behavior, and the protective motivation theory (see for a review Munro, Lewin, Swart, \& Volmink, 2007). In brief, these theories identify the perceived susceptibility and severity of a disease, the perceived costs and benefits of preventive actions, the intention to perform a behavior, and the coping appraisal of the preventive actions as determinants of human responses to a health threat (Bish \& Michie, 2010; Munro et al., 2007). Other theories emphasized the concurrent role of emotions in response to a health threat, such as the parallel processing model and the PRIME theory of motivation (Bish \& Michie, 2010). These theories argue that emotional responses interact with the cognitive appraisal of a health threat and both emotional and cognitive systems determine human behavior in response to a health threat.

However, another element of human cognition that should not be overlooked in face of a health threat is optimism bias. Optimism bias is a pervasive pattern in future projections that refers to our tendency to

\footnotetext{
* Corresponding author at: Behavioural Science Institute, Radboud University, Montessorilaan 3, 6525 HR Nijmegen, the Netherlands.

E-mail addresses: i.fragkaki@pwo.ru.nl (I. Fragkaki), d.maciejewski@pwo.ru.nl (D.F. Maciejewski), e.weijman@pwo.ru.nl (E.L. Weijman), J.Feltes@tudelft.nl (J. Feltes), m.cima@bsi.ru.nl (M. Cima).
} 
overestimate the likelihood of positive events in our future while underestimating the likelihood of negative events (Sharot, 2011). For instance, despite the high rates of divorces or the high rates of medical diseases, individuals tend to believe that the possibility of them getting a divorce is negligible and they are unlikely to suffer from medical diseases in the future. In light of a pandemic, optimism bias can be viewed as the perception of individuals that they will not get infected despite the high rates of infections around the world. Even though optimism is associated with positive health outcomes and success in life (Sharot, 2011), optimism bias can be detrimental in dangerous situations, such as a global pandemic. Individuals who underestimate the risk of getting infected might be less inclined to comply with the NPIs, putting themselves as well as others in danger and contributing to the spread of the virus.

A recent study examined whether optimism bias influenced the engagement in protective behaviors during the Covid-19 pandemic in the United States in March 2020 (Wise, Zbozinek, Michelini, Hagan, \& Mobbs, 2020). They found that individuals underestimated their personal risk of contracting the disease compared to the average person and participants who estimated their personal risk of infection as low, they also engaged less in protective behaviors (Wise et al., 2020). This study supports the presence of optimism bias in face of the current pandemic and its negative effect on engagement in protective behaviors. Another recent study investigated risk perception in 10 countries focusing on perceived severity, perceived likelihood of infection, and feelings of worry (Dryhurst et al., 2020). The results showed that risk perception was high across countries and higher levels of risk perception were positively associated with higher engagement in protective behaviors.

Finally, population adherence to the NPIs is also influenced by people's trust and satisfaction with the government (Bish \& Michie, 2010; Van Bavel et al., 2020). Crucially, trust in government may have distinct effects on population adherence to NPIs and perceived risk. Evidence from previous pandemics showed that trust in government was positively related to engagement in preventive behaviors (Bish \& Michie, 2010) and a recent study showed an increase in satisfaction and trust in the government from pre- to post- lockdown in New Zealand (Sibley et al., 2020). Individuals who trust the government might be confident in its ability to handle the situation appropriately and thus adhere to the imposed measures.

On the other hand, dissatisfaction and lack of trust in the government may be associated with both higher and lower perceived severity and risk as well as both higher and lower compliance with the NPIs. For instance, individuals who perceive the situation to be very severe and are anxious, they might believe that the government's response to the situation is not sufficient and the measures are ineffective. These individuals may be dissatisfied with the government's response and engage more in protective behaviors to protect themselves. Conversely, individuals with high optimism bias may think that the government exaggerates the situation, perceive the situation to be of low risk and feel dissatisfied with the government and the imposed measures.

Based on evidence from previous pandemics and recent studies during the Covid-19 pandemic, the current study examined how optimism bias, feelings of anxiety, and perceived severity of the situation affected people's engagement in protective behavioral changes and their satisfaction with the governments' response to the current pandemic. We also examined whether satisfaction with the governments' response was associated with engagement in behavioral changes. We used an online survey to examine these associations in individuals from different countries (mainly Netherlands, Germany, USA, Greece) during April and May 2020. We hypothesized that optimism bias would be related to lower engagement in protective behaviors, whereas higher anxiety and perceived severity would be associated with higher engagement in protective behavioral changes. We also explored whether optimism bias, anxiety, and perceived severity influenced people's satisfaction with the government. We expected that high levels of optimism bias, anxiety, and perceived severity would be associated with lower satisfaction with the government, albeit for different reasons. Finally, we explored whether satisfaction with the government would influence the engagement in behavioral changes and whether it interacted with perceived severity of the situation. We hypothesized that individuals who perceived the situation to be very severe and were not satisfied with the government's response, would engage more in behavioral changes.

\section{Material and methods}

\subsection{Participants}

The study applied a snowball sampling strategy and recruited 935 participants $\left(M_{\text {age }}=34.29, S D_{\text {age }}=12.43\right)$. The sample includes a higher number of women ( $68.88 \%$ females) and individuals with higher level of education ( $72.41 \%$ of participants have attended university). The participants were living predominantly in the following countries: Netherlands - $63.85 \%$, Greece $-17.97 \%$, Germany $-7.16 \%$, USA $7.38 \%$, other countries (e.g., United Kingdom, Belgium) $-3.64 \%$. The majority of participants lived in their country of origin $(77.54 \%)$. Detailed demographic information are presented in the Supplemental Material Table S1. The survey was distributed in April and May 2020. The majority of responses were recorded in April (91.12\%) and particularly the first two weeks of April (69.09\%). The participants received information about the study at the beginning of the survey and provided informed consent electronically. The study was approved by the Ethics Committee, Faculty of Social Sciences, Radboud University and was conducted in accordance with the Declaration of Helsinki.

\subsection{Instruments}

The survey included questions about emotions, thoughts, and behavioral changes due to Covid-19, opinions about the governments' responses to the current crisis, and demographic characteristics (country of residence, country of origin, age, gender, and education).

\subsubsection{Information about Covid-19}

The participants were asked three questions about Covid-19: "When did you hear about the Covid-19 for the first time?", "Has someone that you know been infected with Covid-19?", "Has someone that you know passed away due to Covid-19?"

\subsubsection{Optimism bias}

The participants completed three items to assess optimism bias: "I don't think it's going to happen to me", "I don't think it's going to happen to my loved ones", "I am afraid that someone I love may get infected" (reverse coded). The items were rated in a 5-point Likert scale ( 1 = strongly disagree, $5=$ strongly agree $)$ and a mean score was computed with higher values indicating higher optimism bias. The Cronbach alpha was 0.65 .

\subsubsection{Anxiety}

The participants were asked questions about feelings of worry and stress about Covid-19 when they first heard about it and now (at the time of survey completion). They also answer questions about their feelings of worry, stress, and fear due to Covid-19 about themselves, their loved ones, people in the country they reside, people from their country of origin, and people around the world in a 5-point Likert scale $(1=$ not at all, $5=$ extremely). A mean score of all the items was computed as an index of overall feelings of anxiety and higher scores indicate higher levels of anxiety. The Cronbach alpha was 0.95 .

\subsubsection{Perceived severity}

The participants completed four items about how serious they perceive the current situation to be. The items were: "I think it is a very severe situation globally", "It is a big problem in the country I reside", "I think it is like the flu" (reverse coded), "I think the situation is 
exaggerated by the media" (reverse coded). The items were rated in a 5point Likert scale $(1=$ strongly disagree, $5=$ strongly agree $)$ and a mean score was computed with higher scores indicating higher perceived severity. The Cronbach alpha was 0.67 .

\subsubsection{Behavioral changes}

The participants completed seven items regarding their behavioral responses to the current situation. The items were: "I stay at home as much as possible", "I wash my hands more often than I used to", "I comply with the measures imposed by the government", "I go for walks to get some fresh air 2 or more times a week" (reverse coded), "I visit or go out with my family at least once a week" (reverse coded), "I visit or go out with my friends at least once a week" (reverse coded), and "I wear a mask when I go outside". The items were rated in a 5-point Likert scale ( 1 = strongly disagree, $5=$ strongly agree $)$ and a mean score was computed with higher scores indicating more engagement in behavioral changes. The Cronbach alpha was 0.62 .

\subsubsection{Opinions about the governments' response}

The participants completed seven items in a 5-point Likert scale $(1=$ strongly disagree, $5=$ strongly agree) about their personal opinions on the response of the government in the country they reside. A scale of satisfaction with the government response was computed with the mean of the following three items: "I think the response of the government is appropriate to the situation", "I think the government reacted fast to the situation", "I think the measures are not as strict as they should be" (reverse coded). The Cronbach alpha was 0.70. Other specific opinions about the governments' response were examined separately: "I think the government prioritizes the economy", "I think the measures are strict", "I think the government listens to the advice of the experts", and "I think the government exaggerates the situation".

\subsection{Procedure}

The survey was conducted using Qualtrics and it was distributed electronically with an anonymized link. A snowball sampling method was used with distribution of the survey in university employees and students, social media, and institutions. No identifiable digital information was recorded. The participants were informed about the study and they provided informed consent electronically. The survey was distributed in four languages: English, Dutch, German, and Greek. The translations and back-translations were performed by the authors with the help of native speakers. The number of participants who completed the total survey was $844(90.27 \%)$. The available data from all the responders $(N=935)$ were included in the study.

\section{Results}

Multiple regression analyses were performed to examine the research questions using $\mathrm{R}$ ( $\mathrm{R}$ Core Team, 2017). From the total sample, $47.59 \%$ of the participants reported that they knew someone who had been infected by Covid-19 and 8.23\% reported that they knew someone who passed away due to Covid-19. Most of the participants heard about the Covid-19 in January (80.32\%), 18.07\% heard about it in February, and $1.06 \%$ heard about it in March.

Table 1

Means and standard deviations of the study variables.

\begin{tabular}{lcc}
\hline & M & SD \\
\hline Anxiety & 2.69 & 0.84 \\
Optimism bias & 2.24 & 0.67 \\
Perceived severity & 3.92 & 0.71 \\
Behavioral changes & 3.56 & 0.59 \\
Satisfaction with government & 3.36 & 0.88 \\
Age & 34.29 & 12.43 \\
\hline
\end{tabular}

Table 1 presents the means and standard deviations of the study variables and Table 2 presents the correlations among these variables. Fig. 1 presents the distributions of the items used to assess optimism bias and perceived severity.

\subsection{Anxiety}

The responders stated that their feelings of anxiety increased from the time they first heard about Covid-19 $(M=1.72, S D=0.81)$ to the time of survey completion $(M=2.99, S D=0.90), t(877)=-39.49, p<$ $.001,95 \% \mathrm{CI}[-1.34,-1.21]$. The responders reported higher levels of anxiety due to Covid-19 for their loved ones $(M=3.21, S D=1.01)$ than themselves $(M=2.11, S D=0.95), t(877)=-36.70, p<.001,95 \% \mathrm{CI}$ $[-1.15,-1.04]$. They also reported higher levels of anxiety due to Covid-19 for other people $(M=2.71, S D=0.94)$ than themselves $(M=$ $2.11, S D=0.95), t(877)=-20.13, p<.001,95 \%$ CI $[-0.66,-0.54]$.

The regression analysis demonstrated that higher levels of overall anxiety were reported by older responders, $b=0.01, S E=0.002, t(868)$ $=2.30, p=.02$, by females compared to males, $b=0.25, S E=0.06, t$ $(868)=4.23, p<.001$, and by responders who knew someone that passed away due to Covid-19, $b=-0.23, S E=0.10, t(868)=-2.26, p=$ .02 . Educational level was not significantly associated with anxiety $(p>$ .05). Satisfaction with the government was negatively associated with anxiety, $b=-0.20, S E=0.03, t(868)=-6.57, p<.001$.

\subsection{Optimism bias}

It is noteworthy that $13.26 \%$ of the responders reported that they thought they would not get infected. Levels of optimism bias were higher in males than females, $b=-0.19, S E=0.05$, $t(855)=-3.66, p<$ .001 , and in responders who did not know anyone that passed away due to Covid-19, $b=0.34, S E=0.09, t(855)=3.94, p<.001$. Age and educational level were not significantly associated with optimism bias ( $p$ s $>0.05$ ). Satisfaction with the government was negatively associated with optimism bias, $b=-0.06, S E=0.03, t(868)=-2.07, p=.04$.

\subsection{Perceived severity of the situation}

Higher perceived severity was reported by females compared to males, $b=0.14, S E=0.05, t(855)=2.68, p=.007$, and by responders who knew someone who was infected by Covid-19, $b=-0.10, S E=$ $0.05, t(855)=-2.06, p=.04$. Age and educational level were not significantly associated with perceived severity of the situation ( $p s>$ $0.05)$. Satisfaction with the government was not significantly associated with perceived severity, $b=0.05, S E=0.03, t(868)=1.78, p=.08$.

\subsection{Engagement in behavioral changes}

We performed a regression analysis to examine whether optimism bias, perceived severity of the situation, feelings of anxiety, and satisfaction with the government's response predicted the engagement in behavioral changes. We also examined the interaction between satisfaction with government's response and perceived severity in predicting behavioral changes. We included age, gender, date of survey completion, country of residence, education level, and whether the participants knew someone who has been infected or passed away due to Covid-19 as control variables. Fig. 2 presents the distributions of the behavioral changes measured.

The regression analysis demonstrated that individuals with higher levels of optimism bias engaged less in behavioral changes, $b=-0.09$, $S E=0.03, t(834)=-3.23, p=.001)$, whereas individuals with higher levels of perceived severity, $b=0.24, S E=0.03, t(834)=9.28, p<.001$, and higher levels of anxiety, $b=0.14, S E=0.02, t(834)=6.21, p<.001$, engaged more in behavioral changes. The total variance explained was $43 \%$.

In addition, government satisfaction was significantly related to 
Table 2

Correlations Among the Study Variables.

\begin{tabular}{|c|c|c|c|c|c|c|}
\hline & Anxiety & Optimism bias & Perceived severity & Behavioral changes & Satisfaction with government & Age \\
\hline Anxiety & 1 & & & & & \\
\hline Optimism bias & $-0.36^{* *}$ & 1 & & & & \\
\hline Perceived severity & $0.23^{* *}$ & $-0.39 * *$ & 1 & & & \\
\hline Behavioral changes & $0.47^{* *}$ & $-0.27^{* *}$ & $0.32^{* *}$ & 1 & & \\
\hline Satisfaction with government & $-0.14 * *$ & -0.01 & $-0.09^{* *}$ & $-0.17^{* *}$ & 1 & \\
\hline Age & $0.08^{*}$ & $0.13^{* *}$ & -0.04 & $0.08^{*}$ & 0.06 & 1 \\
\hline
\end{tabular}

* $p<.05$.

$p<.01$.

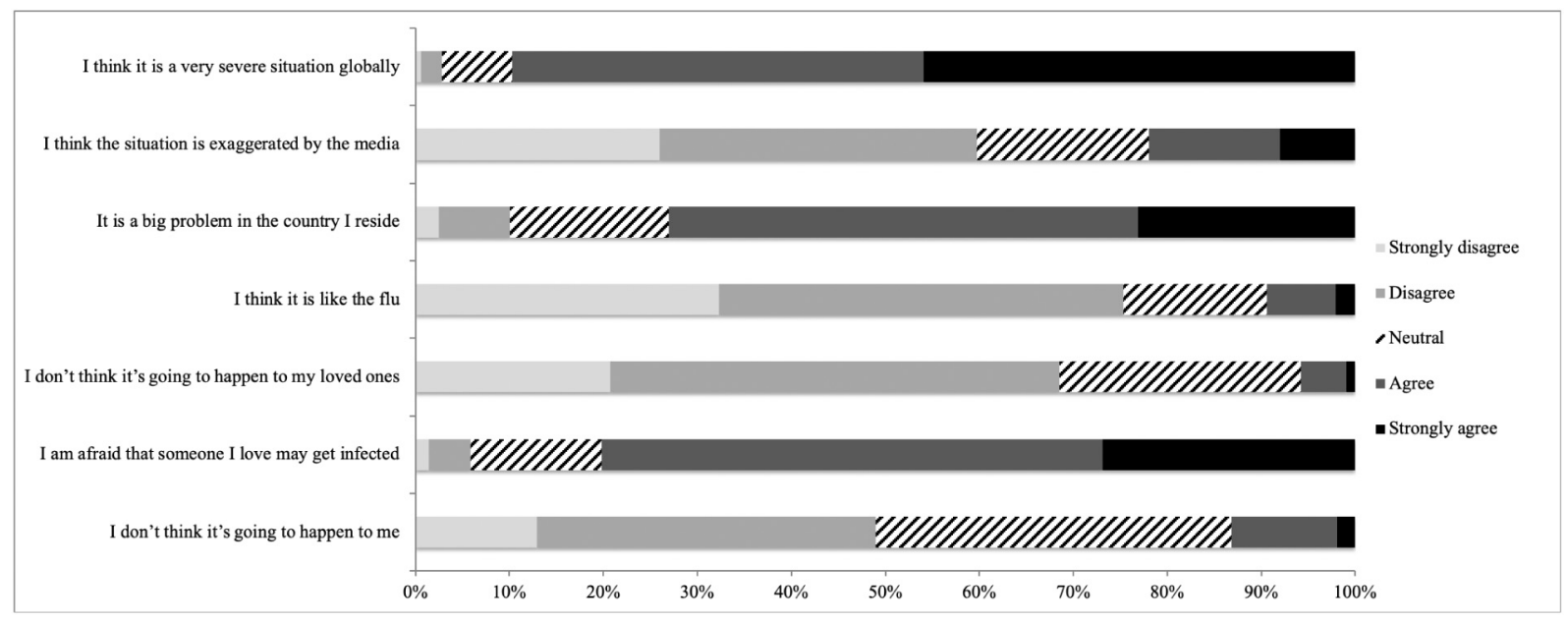

Fig. 1. The distributions of the items for optimism bias and perceived severity.

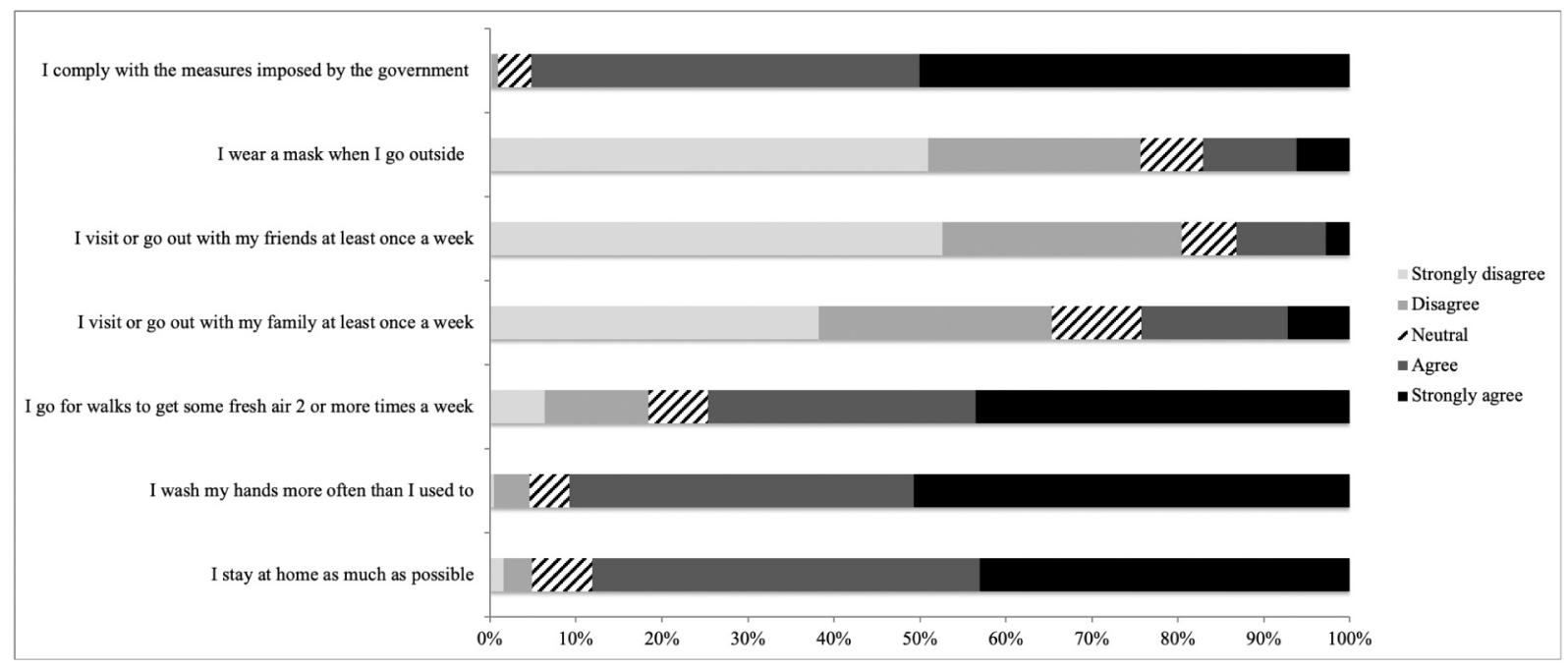

Fig. 2. The distributions of behavioral changes.

engagement in behavioral changes, $b=-0.09, S E=0.02, t(834)=$ $-4.21, p<.001$, indicating that individuals who were less satisfied with government's response, engaged more in behavioral changes. The interaction between perceived severity and government satisfaction was significant, $b=-0.07, S E=0.03, t(834)=-2.81, p=.005$. A simple slope analysis showed that participants who reported low perceived severity and low government satisfaction engaged the least in behavioral changes, whereas participants who reported high perceived severity and low government satisfaction engaged the most in behavioral changes. Fig. 3 illustrates the interaction.

\subsection{Satisfaction with government's response}

Fig. 4 presents the distributions of the items about the government's response. The analyses showed that both higher levels of optimism bias, $b=-0.09, S E=0.05, t(836)=-2.07, p=.04$, and higher levels of anxiety, $b=-0.24, S E=0.04, t(836)=-6.57, p<.001$, predicted lower satisfaction. There was a trending positive association between perceived severity and satisfaction, $b=0.08, S E=0.04, t(836)=1.78, p$ $=.08$. The total variance explained was $32 \%$.

We also examined other specific opinions about the government's responses. We found that individuals with higher levels of optimism bias 


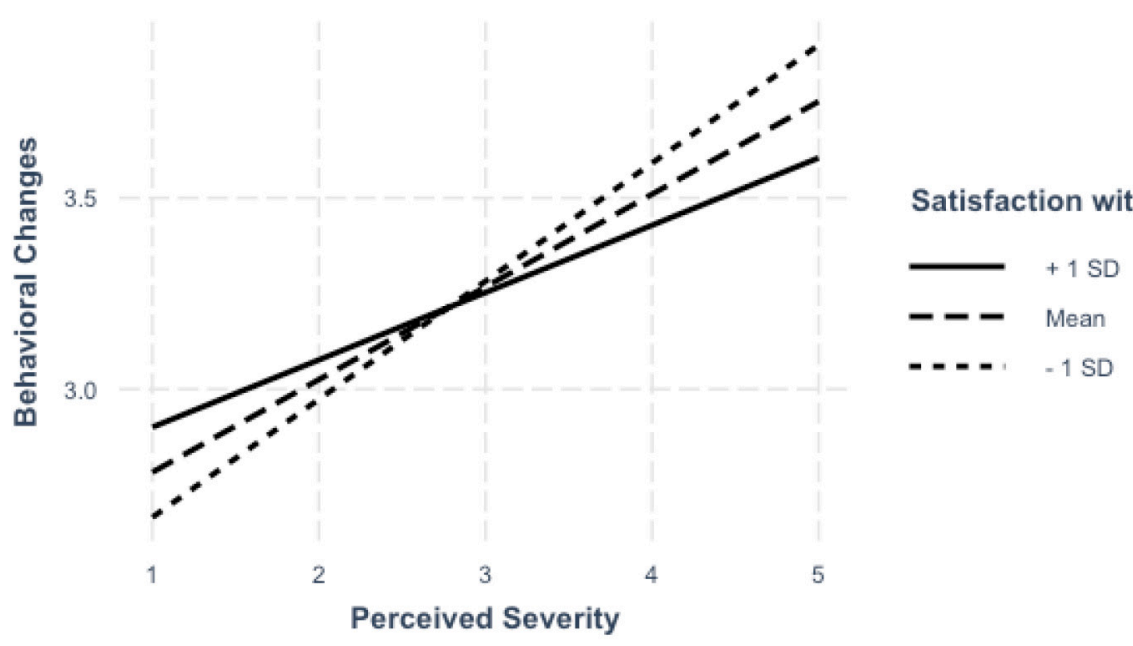

Fig. 3. The Interaction Between Perceived Severity and Government Satisfaction on Engagement in Behavioral Changes.

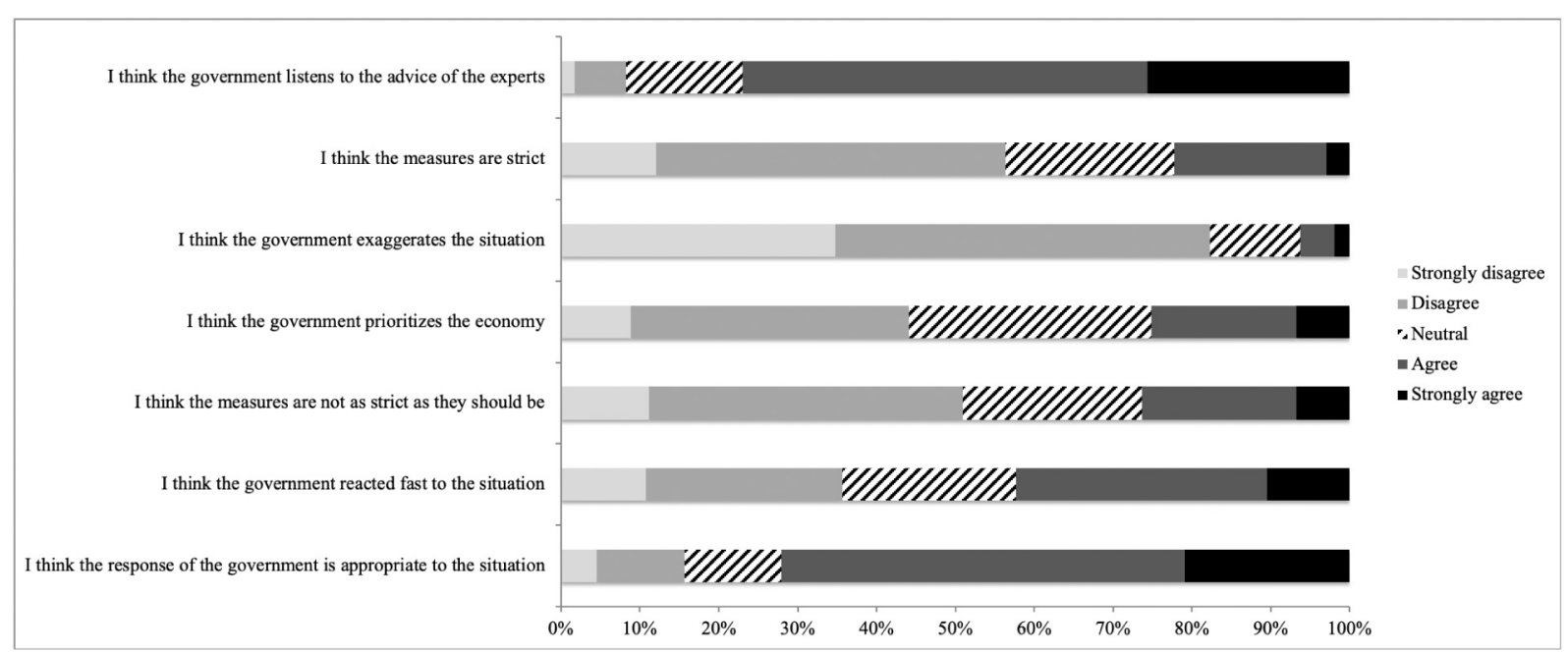

Fig. 4. The distributions of the items about government's response.

were more likely to consider the measures strict, $b=0.13, S E=0.06, t$ $(836)=2.26, p=.02$, whereas individuals with higher levels of perceived severity, $b=-0.22, S E=0.06, t(836)=-3.99, p<.001$, and higher levels of anxiety, $b=-0.12, S E=0.05, t(836)=-2.37, p=.02$, were less likely to consider the measures strict.

Furthermore, individuals with higher levels of anxiety were more likely to believe that the government prioritizes the economy, $b=$ $-0.37, S E=0.05, t(836)=7.46, p<.001$. In contrast, individuals with higher levels of perceived severity were more likely to disagree with this statement, $b=-0.15, S E=0.06, t(836)=-2.57, p=.01$. Optimism bias was not significantly linked to this statement, $b=0.09, S E=0.06, t$ $(836)=1.47, p=.14$.

Individuals with higher levels of optimism bias, $b=-0.12$, $S E=$ $0.05, t(836)=2.37, p=.02$, and high levels of anxiety, $b=-0.22, S E=$ $0.04, t(836)=-5.57, p<.001$, were more likely to disagree with the statement "I think the government listens to the advice of the experts". In contrast, individuals with high perceived severity were more likely to agree with this statement, $b=0.28, S E=0.05, t(836)=6.18, p<.001$.

Individuals with higher levels of optimism bias, $b=0.15, S E=0.04, t$ $(836)=3.35, p<.001$, believed that the government exaggerates the situation, whereas higher levels of perceived severity was negatively related to this statement, $b=-0.64, S E=0.04, t(836)=-15.60, p<$ .001 .

\section{Discussion}

The aim of the study was to investigate whether optimism bias, anxiety, and perceived severity of the situation affected people's engagement in protective behaviors and their satisfaction with the governments' response during the current pandemic.

The first interesting finding is the role of optimism bias. Individuals with high levels of optimism bias engaged less in protective behavioral changes. This is in line with the study by Wise et al. (2020) that also found an association between optimism bias and lower engagement in protective behaviors. Optimism bias might be particularly detrimental during a pandemic, as individuals tend to underestimate the possibility of contracting the virus and our data suggest that these individuals engaged less in protective behavioral changes. As a result, they might have a higher chance of getting infected and contribute to the spread of the coronavirus. We thus argue that optimism bias should be taken into account by policymakers especially in information dissemination, as optimism bias is not easily eliminated even when we are presented with credible information that contradicts our bias (Sharot, 2011).

Additionally, individuals with high levels of optimism bias were less satisfied with the response of the government. A closer look at specific opinions about the government revealed that individuals with high optimism bias were more likely to consider the imposed measures strict and more likely to believe that the government does not listen to the 
advice of the experts, as well as exaggerates the situation. Taken together, we observe that individuals with higher optimism bias have a profile of lower perceived severity of the situation, lower risk perception, dissatisfaction with the government, and less engagement in protective behaviors.

The second finding is the role of anxiety and perceived severity of the situation. Individuals with high levels of anxiety and those who consider the situation serious engaged more in protective behavioral changes. This is also in line with a recent study (Dryhurst et al., 2020) as well as evidence from previous pandemics (Bish \& Michie, 2010). High levels of anxiety may also lead to negative outcomes, such as complete isolation, obsessive engagement in protective behaviors, and mental health problems. As also suggested by West, Michie, Rubin, and Amlôt (2020) the policymakers, the media, and the authorities should carefully design the way they communicate the progress of the pandemic, the scientific evidence, and the necessity of NPIs taking into account our individual differences. People with high optimism bias might need more convincing and substantiated arguments to better understand the severity of the situation, while others might also need messages aiming to reduce their anxiety.

In relation to satisfaction with the government, individuals with high levels of anxiety reported lower levels of satisfaction. In particular, anxious individuals thought that the measures were not strict and that the government prioritizes the economy. Moreover, anxious individuals believed that the government does not listen to the advice of the experts. Interestingly, individuals with high optimism bias also agreed with this statement. In contrast, individuals with high perceived severity were more likely to believe that the government listens to the advice of the experts. This is an interesting observation that highlights the subjective interpretation of information. Policymakers argue that the enforcement of NPIs is based on scientific evidence, but it seems that whether citizens are convinced of that might depend on different perceptions and individual differences.

The third important finding of this study was the interaction between perceived severity and government satisfaction on the level of engagement in protective behavioral changes. The responders who were less likely to believe that the situation is serious and were less satisfied with the response of the government, engaged the least in protective behavioral changes. These individuals might believe that the NPIs are excessive and unnecessary and that the government does not handle the situation appropriately and thus they do not engage as much in protective behaviors. In contrast, responders who were more likely to believe that the situation is very serious and they were less satisfied with the response of the government engaged the most in protective behavioral changes. These individuals might blame the government for not imposing stricter NPIs and feel that the existing measures are not sufficient to protect the citizens and thus engage more in protective behaviors in an effort to protect themselves.

To better understand the generalizability and the context of the findings, several limitations should be acknowledged. First, the sample of the study was recruited with the snowball sampling strategy and it is not representative of the population. Specific characteristics of the sample that need to be underscored are the higher number of female participants, participants with higher level of education, and participants living in the Netherlands. Second, the number of participants from other countries was considerably lower than the number of participants from the Netherlands, impeding a rigorous comparison between countries. Considering the different governments' responses to the pandemic among countries, it is reasonable to expect different behavioral changes and opinions between citizens of different countries. For instance, participants living in the Netherlands engaged less in behavioral changes compared to participants living in Greece, but we can assume that this difference is driven by the different NPIs applied in the two countries in April and May 2020. Greece implemented a national lockdown in March that required many behavioral changes, whereas the Netherlands imposed less strict NPIs. It is important to conduct large-scale studies that can provide information on how psychological factors influence human responses to the pandemic among various countries.

Third, this study provides cross-sectional data and thus no causal effects can be determined. For instance, we found an association between high optimism bias and lower engagement in protective behavioral changes, but we cannot conclude whether optimism bias caused the lower level of engagement. Fourth, we focused on optimism bias, anxiety, and perceived severity, but there are numerous other factors that might influence people's response to the pandemic and their opinion on government's response, such as social norms, moral thinking, personality characteristics, motivational processes, and leadership style of authorities (Van Bavel et al., 2020; West et al., 2020).

Taken together, the findings suggest that optimism bias, anxiety, and perceived severity of the situation play a role in how we interpret the information we receive, how we shape our opinions about the response of the government, and ultimately how we react to the situation. Given that the successful containment of the coronavirus depends highly on population adherence to the NPIs, it is crucial to unravel the psychological factors that influence people's responses to NPIs. It is imperative for policymakers to better understand which factors influence population adherence to the NPIs in order to implement more effective policies and make better predictions on population response over time. Finally, as also suggested by West et al. (2020), the policymakers should carefully design the way they communicate the progress of the pandemic and the necessity of NPIs taking into account different communication needs and subjective perceptions.

Supplementary data to this article can be found online at https://doi. org/10.1016/j.paid.2021.110781.

\section{CRediT authorship contribution statement}

Iro Fragkaki: Conceptualization, Methodology, Formal analysis, Investigation, Data curation, Writing - original draft, Visualization, Project administration. Dominique F. Maciejewski: Investigation, Writing - review \& editing. Esther L. Weijman: Investigation, Writing review \& editing. Jonas Feltes: Investigation, Writing - review \& editing. Maaike Cima: Conceptualization, Writing - review \& editing, Supervision.

\section{Declaration of competing interest}

None.

\section{Acknowledgements}

The authors would like to thank Themistoklis Mavridis and Ramon Daniël Kleiss for their help with the translations.

\section{References}

Balinska, M., \& Rizzo, C. (2009). Behavioural responses to influenza pandemics: What do we know? PLoS Currents, 1. https://doi.org/10.1371/currents.rm1037.

Bish, A., \& Michie, S. (2010). Demographic and attitudinal determinants of protective behaviours during a pandemic: A review. British Journal of Health Psychology, 15(4), 797-824. https://doi.org/10.1348/135910710X485826.

R Core Team, 2017. R: A language and environment for statistical computing. https:// www.R-project.org/.

Dryhurst, S., Schneider, C. R., Kerr, J., Freeman, A. L., Recchia, G., Van Der Bles, A. M., .. van der Linden, S. (2020). Risk perceptions of COVID-19 around the world. Journal of Risk Research, 1-13. https://doi.org/10.1080/13669877.2020.1758193.

Hale, T., Webster, S., Petherick, A., Phillips, T., \& Kira, K. (2020). Oxford COVID-19 government response tracker. Blavatnik school of government. Data use policy: Creative commons attribution CC BY standard. Retrieved from https://covidtracker.bsg.ox. ac.uk/.

Munro, S., Lewin, S., Swart, T., \& Volmink, J. (2007). A review of health behaviour theories: How useful are these for developing interventions to promote long-term medication adherence for TB and HIV/AIDS? BMC Public Health, 7(1), 1-16. https:// doi.org/10.1186/1471-2458-7-104.

Sharot, T. (2011). The optimism bias. Current Biology, 21(23), R941-R945. https://doi. org/10.1016/j.cub.2011.10.030. 
Sibley, C. G., Greaves, L. M., Satherley, N., Wilson, M. S., Overall, N. C., Lee, ... Barlow, F. K. (2020). Effects of the COVID-19 pandemic and nationwide lockdown on trust, attitudes towards government, and wellbeing. American Psychologist, 75(5), 618-630. https://doi.org/10.1037/amp0000662.

Van Bavel, J. J., Baicker, K., Boggio, P. S., Capraro, V., Cichocka, A., Cikara, M., ... Drury, J. (2020). Using social and behavioural science to support COVID-19 pandemic response. Nature Human Behaviour, 1-12. https://doi.org/10.1038/ s41562-020-0884-Z.
West, R., Michie, S., Rubin, G. J., \& Amlôt, R. (2020). Applying principles of behaviour change to reduce SARS-CoV-2 transmission. Nature Human Behaviour, 1-9. https:// doi.org/10.1038/s41562-020-0887-9.

Wise, T., Zbozinek, T. D., Michelini, G., Hagan, C. C., \& Mobbs, D. (2020). Changes in risk perception and protective behavior during the first week of the COVID-19 pandemic in the United States. Royal Society Open Science, 7(9). https://doi.org/10.1098/ rsos. 200742 . 